\title{
An Efficient and Simple Analytical Model for Analysis of Propagation Properties in Impedance Waveguides
}

\author{
Olli Luukkonen, Constantin R. Simovski, Antti V. Räisänen, and Sergei A. Tretyakov,
}

\begin{abstract}
In this paper propagation properties of a parallel-plate waveguide with tunable artificial impedance surfaces as sidewalls are studied both analytically and numerically. The impedance surfaces comprise an array of patches over a dielectric slab with embedded metallic vias. The tunability of surfaces is achieved with varactors. Simple design equations for tunable artificial impedance surfaces as well as dispersion equations for the TE and TM modes are presented. The propagation properties are studied in three different regimes: a multi-mode waveguide, a single-mode waveguide, and below-cutoff waveguide. The analytical results are verified with numerical simulations.
\end{abstract}

\section{Index Terms}

high-impedance surface, tunable, impedance waveguide, propagation properties

\section{INTRODUCTION}

Artificial impedance surfaces [1]-[9] have received a lot of interest since the beginning of the last decade. In general the artificial impedance surfaces are composed of a capacitive grid on top of a thin metal-backed dielectric substrate. The substrate may include vias, as in [2], [4], or may not include them ( [9]-[11]). Nevertheless, the purpose in both types of designs is to use the grounded substrate to provide an inductive response that together with the capacitive grid would create a resonant structure. Because of the resonant nature such impedance surfaces are commonly referred to as high-impedance surfaces.

Recently some research has been devoted to electrical tunability of high-impedance surfaces. The tunability has been realized e.g. by connecting adjacent patches or strips to each other by voltage controllable varactors in order to construct tunable antennas [12], [13], phase shifters [14], lenses [15], band-pass filters [16], and band-stop filters [17]. In addition to the varactor-based tunable impedance surfaces also a MEMS-based tunable high-impedance surface has been proposed in [18]. In this paper we concentrate on the varactor-based tunable high-impedance surfaces.

In [12]-[15] the analysis of the tunable high-impedance surface has been done by using a simplistic lumped-element model of the surface, similar to that of [19]. In addition to the lumped element models, also a layered homogeneous material models have been developed [20], [21] to predict the behavior of non-tunable high-impedance surfaces. The lumped-element model [19] offers guidelines for the design of a tunable high-impedance surface. For instance, in [22] an equivalent circuit model is used to approximate the effect of the varactor resistance to the reflection characteristic of a high-impedance surface similar to that described in [12]. However, the lumped-element model cannot be used for an accurate analysis of the surface nor for the analysis of many applications because it does not take the oblique incidence into account.

The work was supported in part by the Academy of Finland and Tekes through the Center-of-Excellence Programme. Olli Luukkonen wishes to thank the Nokia Foundation for financial support.

O. Luukkonen, C. R. Simovski, A. V. Räisänen, and S. A. Tretyakov are with are with the Department of Radio Science and Engineering/SMARAD Center of Excellence, TKK Helsinki University of Technology, P.O. 3000, FI-02015 TKK, Finland (email: olli.luukkonen@tkk.fi) 
Because of their unique characteristics the artificial impedance surfaces have been used as wall coatings in different waveguiding structures. For instance, close to the resonance frequency the input surface impedance of an artificial impedance surface is high and the surface behaves as a magnetic conductor. This feature has been utilized in quasi-TEM waveguides [11], [23]-[25]. Furthermore, the possibility to electrically vary the input surface impedance of an artificial impedance has been exploited in many waveguiding applications [14]-[17]. For the design of such applications accurate knowledge about the waveguide modes is essential. In [26] analysis of dispersion in a rectangular waveguide with impedance sidewalls comprising non-tunable dipole-like frequency selective surfaces (FSS) on a metal-backed dielectric slab has been done numerically by using the method of moments.

In this paper we introduce a simple analytical model for a varactor-tunable high-impedance surfaces that predicts the response of the impedance surface very well even for oblique incidences. This model is general and can be used for surfaces that comprise any type of a rectangular patch array, for instance for such as those in [12], [13]. Together with the dispersion equations, the model for the tunable impedance surfaces is used to study the propagation properties of a parallel-plate waveguide having either one or two tunable impedance surfaces replacing the metallic plates. In particular, parallel-plate waveguides allowing multimode or single-mode propagation are studied. Also a waveguide operating below its cutoff frequency is considered. Numerical full-wave simulations verify the analytical results and show that the used analytical model describes accurately the properties of tunable impedance surfaces in waveguiding set-ups.

\section{ANALYTICAL MODEL}

We study the propagation properties of a parallel-plate waveguide having tunable impedance surfaces using the plane-wave interpretation. The two-dimensional waveguide geometry is illustrated in Fig. 1 , The number of the transversal wave numbers is limited to one as no propagation takes place in the $x$-direction. The waveguide is confined in the $y$-direction by plates that can be modeled with an impedance surface $Z_{\text {inp }}^{+}$ or $Z_{\text {inp }}^{-}$that are dependent both on the wave number $k$ and the propagation constant along the waveguide $\beta$. The notation \pm refers to the upper/lower surface, respectively.

The dispersion relations for the parallel-plate waveguide presented in Fig. 1 can be solved from the boundary conditions at the waveguide sidewalls and from the transverse components of the magnetic and electric fields. The transverse components can be calculated from the longitudinal $(z-)$ components using general plane-wave solutions in the $y$-dimension (see e.g. [27]). The resulting dispersion equation for the TE modes can be written as:

$$
\tan \left(k_{\mathrm{y}} d\right)=j \eta \frac{k}{k_{\mathrm{y}}} \frac{Z_{\mathrm{inp}}^{+}+Z_{\mathrm{inp}}^{-}}{\eta^{2} \frac{k^{2}}{k_{\mathrm{y}}^{2}}+Z_{\mathrm{inp}}^{+} Z_{\mathrm{inp}}^{-}} .
$$

For TM modes the dispersion equation reads

$$
\tan \left(k_{\mathrm{y}} d\right)=j \eta \frac{k_{\mathrm{y}}}{k} \frac{Z_{\mathrm{inp}}^{+}+Z_{\mathrm{inp}}^{-}}{\eta^{2} \frac{k_{\mathrm{y}}^{2}}{k^{2}}+Z_{\mathrm{inp}}^{+} Z_{\mathrm{inp}}^{-}} .
$$

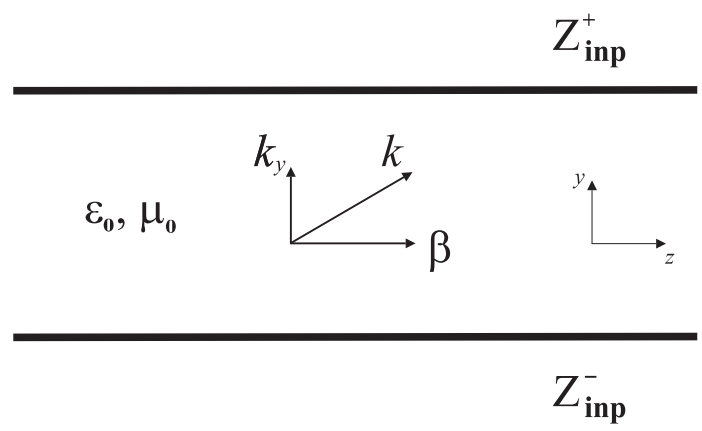

Fig. 1. Illustration of the two dimensional waveguide confined by two impedance surfaces. 


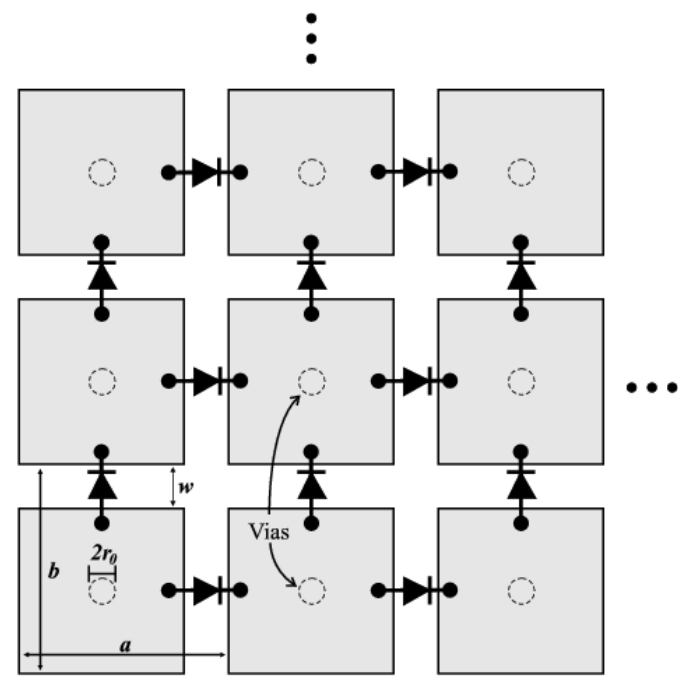

(a)

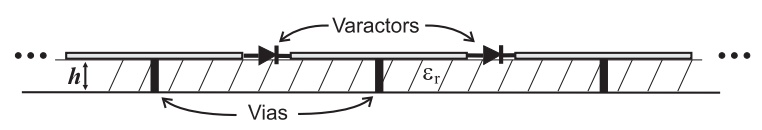

(b)

Fig. 2. (a) A view from the top of the Sievenpiper mushroom structure loaded with varactors. (b) A view from the side.

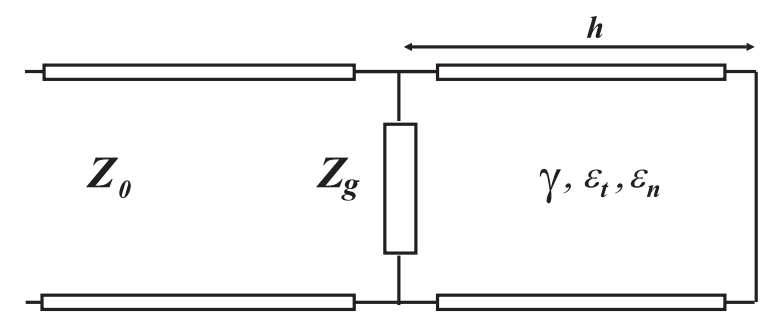

Fig. 3. A transmission-line model for modeling artificial impedance surfaces. $Z_{0}$ is the free-space impedance.

In the above formulae $k_{\mathrm{y}}= \pm \sqrt{k^{2}-\beta^{2}}$ is the transverse wave number, and $\eta$ is the plane-wave impedance of the medium filling the waveguide. By choosing the "minus"-branch of the transverse wave number, also surface modes on the waveguide sidewalls are predicted by the above dispersion equations.

\section{A. The input surface impedance}

The dispersion equations (11) and (2) have been derived for arbitrary surface impedances. In this paper the tunable version of a mushroom-type artificial impedance surface (see Fig. 2), proposed in [2], is studied as a possible particular realization. The metallic plates of a waveguide are replaced with artificial impedance surfaces that are comprised of metallic rectangular patches and metal-backed dielectric substrates with embedded vias. The tunability is achieved by connecting the adjacent rectangular patches to each other by varactors. The input surface impedance of an artificial impedance surface can be modeled through a transmission-line model shown in Fig. 3. The input impedance is hence a parallel connection of the grid impedance of an array of rectangular patches and the surface impedance of a metal-backed dielectric slab with embedded vias:

$$
Z_{\text {inp }}^{-1}=Z_{\mathrm{g}}^{-1}+Z_{\mathrm{s}}^{-1}
$$

In the above equation subscript $g$ refers to the grid impedance of an array of rectangular patches and $s$ refers to the surface impedance of the substrate.

A simple and accurate analytical model for a non-tunable mushroom-type impedance surface is available [7]. In [7] the mushroom structures comprised arrays of patches on top of a dielectric layer. However, in this paper we consider mushroom structures that comprise arrays of patches on top of a metal-backed dielectric slab with embedded metallic vias. The vias are needed to provide the bias voltage for the 
varactors that are used to vary the capacitance between the adjacent strips or patches (as in [12], [14]). For TE modes the electric field component is perpendicular to the vias and, in the case of thin vias, does not excite them. It can be concluded that the analytical model [7] can be readily applied for TE modes in the case of embedded vias by taking the effect of varactors into account correctly. However, for TM modes the electric field has a parallel component to the vias. In this case it is possible to model the metallic vias in a dielectric slab as an effective wire medium [3], [5].

\section{B. Surface impedance of the dielectric slab with embedded vias}

The surface impedance for a wire medium comprising thin perfectly conducting wires reads [5]:

$$
Z_{\mathrm{s}}^{\mathrm{TM}}=j \omega \mu_{0} \frac{\tan \left(\gamma_{\mathrm{TM}} h\right)}{\gamma_{\mathrm{TM}}} \frac{k^{2}-\beta^{2}-k_{\mathrm{p}}^{2}}{k^{2}-k_{\mathrm{p}}^{2}},
$$

where $k=k_{0} \sqrt{\varepsilon_{\mathrm{r}}}$ is the wave number in the host medium,

$$
\gamma_{\mathrm{TM}}^{2}=\omega^{2} \varepsilon_{0} \varepsilon_{\mathrm{t}} \mu_{0}-\frac{\varepsilon_{\mathrm{t}}}{\varepsilon_{\mathrm{n}}} \beta^{2},
$$

$\varepsilon_{\mathrm{t}}$ is the relative permittivity for the fields along the transverse plane, and

$$
k_{\mathrm{p}}=\frac{1}{a \sqrt{\frac{1}{2 \pi} \ln \frac{a^{2}}{4 r_{0}\left(a-r_{0}\right)}}} .
$$

Furthermore, $a$ is the period of the wires, $r_{0}$ is the radius of the wires, and the relative permittivity for the fields along the normal of the medium reads

$$
\varepsilon_{\mathrm{n}}=\varepsilon_{\mathrm{t}}\left(1-\frac{k_{\mathrm{p}}^{2}}{k^{2} \varepsilon_{\mathrm{t}}}\right) .
$$

In the case when the vias are thin and vertically oriented, the relative permittivity for the fields along the transversal plane, $\varepsilon_{\mathrm{t}}$, equals to the relative permittivity of the host medium, $\varepsilon_{\mathrm{r}}$.

For TE modes the electric field is perpendicular to the thin metallic wires. In this case, as discussed above, the electric field does not excite the wires, and the surface impedance for the TE mode is that of a metal-backed dielectric slab (see, e.g., [5]):

$$
Z_{\mathrm{s}}^{\mathrm{TE}}=j \omega \mu_{0} \frac{\tan \left(k_{\mathrm{y}} \sqrt{\varepsilon_{\mathrm{r}}} h\right)}{k_{\mathrm{y}} \sqrt{\varepsilon_{\mathrm{r}}}} .
$$

\section{The grid impedance}

The grid impedance for an array of patches can be calculated through the approximative Babinet principle using the averaged boundary conditions for a mesh of wires or strips. The averaged boundary condition for a mesh of strips is available e.g., in [5]. The grid impedance for an array of ideally conducting patches on top of a dielectric substrate reads [7]:

$$
\begin{gathered}
Z_{\mathrm{g}}^{\mathrm{TM}}=-j \frac{\eta_{\mathrm{eff}}}{2 \alpha}, \\
Z_{\mathrm{g}}^{\mathrm{TE}}=-j \frac{\eta_{\mathrm{eff}}}{2 \alpha\left(1-\frac{k_{0}^{2}}{k_{\mathrm{eff}}^{2}} \frac{\sin ^{2} \theta}{1+\frac{b}{a}} \frac{b}{a}\right)},
\end{gathered}
$$

where the effective wave impedance $\eta_{\text {eff }}=\frac{\eta_{0}}{\sqrt{\varepsilon_{\text {eff }}}}$, the effective wave number $k_{\text {eff }}=k_{0} \sqrt{\varepsilon_{\text {eff }}}, \sin ^{2}(\theta)=$ $\frac{k_{0}^{2}-k_{y}^{2}}{k_{0}^{2}}$, and $b$ and $a$ are the dimensions of the unit cell of the structure along $x-$ and $z$-axis, respectively. 
Further, $\alpha$ is the grid parameter:

$$
\alpha=\frac{k_{\mathrm{eff}} b}{\pi} \ln \left(\frac{1}{\sin \left(\frac{\pi w}{2 b}\right)}\right),
$$

where $w$ is the gap between the adjacent patches (see Fig. 2). The limitations for above expressions of the grid impedance have been discussed in more detail in [7]. It can be concluded here that (9) and (10) are valid when $w<a, b$ and up to the frequencies when $a, b \approx \frac{\lambda}{2}$. The effective relative permittivity for the array of patches or grid of strips on the boundary between two medium having relative permittivities of $\varepsilon_{1}$ and $\varepsilon_{2}$ reads approximately [28]:

$$
\varepsilon_{\mathrm{eff}}=\frac{\varepsilon_{1}+\varepsilon_{2}}{2}
$$

In this paper the array of patches is located on a boundary between free space and wire medium. From above it is known that in a wire medium the fields along the transversal and normal axis see different effective relative permittivities. For an array of patches the electric fields are concentrated mainly between the adjacent patches, transverse with respect to the vias, and the effect of the vias on the electric response is weak. For this reason the transversal relative permittivity of the wire medium is used in (12).

The grid impedances (9) and (10) can be written in a lumped-element form as:

$$
Z_{\mathrm{g}}^{\mathrm{TM}, \mathrm{TE}}=\frac{1}{j \omega C_{\mathrm{g}}^{\mathrm{TM}, \mathrm{TE}}},
$$

where $C_{\mathrm{g}}^{\mathrm{TM}, \mathrm{TE}}$ is the grid capacitance for the TM- or TE-polarized incidence fields. The grid capacitance equals to the averaged capacitance per one unit cell in the $x$ - and $z$-direction for the TM- and TEpolarized cases. Using (9), (10), and (13) the grid capacitance for an array of patches can be written as:

$$
\begin{gathered}
C_{\mathrm{g}}^{\mathrm{TM}}=\frac{b \varepsilon_{0}\left(\varepsilon_{1}+\varepsilon_{2}\right)}{\pi} \ln \left(\frac{1}{\sin \left(\frac{\pi w}{2 b}\right)}\right), \\
C_{\mathrm{g}}^{\mathrm{TE}}=\frac{b \varepsilon_{0}\left(\varepsilon_{1}+\varepsilon_{2}\right)}{\pi} \ln \left(\frac{1}{\sin \left(\frac{\pi w}{2 b}\right)}\right)\left(1-\frac{k_{0}^{2}}{k_{\mathrm{eff}}^{2}} \frac{\sin ^{2} \theta}{1+\frac{b}{a}} \frac{b}{a}\right) .
\end{gathered}
$$

The above formulas for the capacitive grid impedances hold for ideally conducting patches. Although the capacitive impedance is derived through averaged fields on the grid, we may consider a lumpedelement model here. This way the capacitance of the varactors, that are used for tuning, can be included in the analysis easily: The additional capacitance of the varactors is connected in parallel with the grid capacitance and the total impedance of a unit cell is thus a parallel connection of these impedances. Hence, the total grid capacitance of the array of patches with varactors can be written as:

$$
C_{\mathrm{tot}}^{\mathrm{TM}, \mathrm{TE}}=C_{\mathrm{g}}^{\mathrm{TM}, \mathrm{TE}}+C_{\mathrm{var}},
$$

where $C_{\mathrm{g}}$ is the grid capacitance for an array of ideally conducting patches and $C_{\mathrm{var}}$ is the capacitance of the tunable varactor. Finally, the total grid impedance reads:

$$
Z_{\mathrm{g}}^{\mathrm{TM}, \mathrm{TE}}=\frac{1}{j \omega C_{\mathrm{tot}}^{\mathrm{TM}, \mathrm{TE}}}=\frac{1}{j \omega\left(C_{\mathrm{g}}^{\mathrm{TM}, \mathrm{TE}}+C_{\mathrm{var}}\right)} .
$$

In order to most effectively tune the grid impedance, the capacitance of the varactor needs obviously to be considerably larger than the grid capacitance.

The effect of the varactor resistance to the performance of a tunable high-impedance surface has been studied in [22]. It is possible to include the effect of the intrinsic diode resistance to the above analysis in a similar way as done in [22]. However, this is considered to be out of the scope of this paper. 


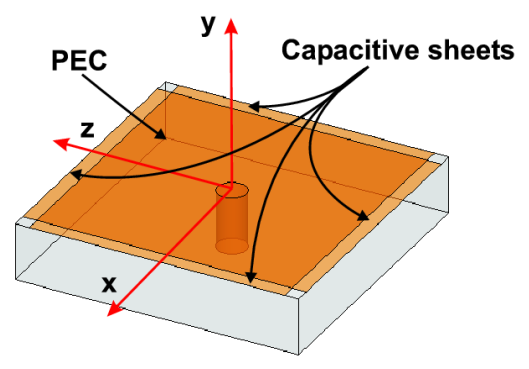

Fig. 4. Color online. Simulation model of the tunable high-impedance surface unit cell. Because of the periodicity, the capacitance of each capacitive sheet equals to $2 C_{\mathrm{var}}$.

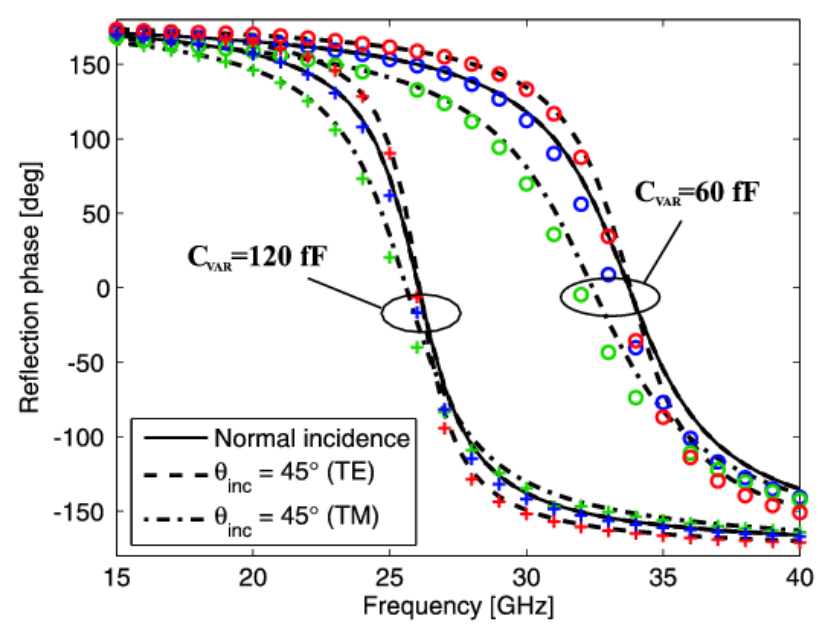

Fig. 5. Color online. The reflection phases for different incidences and for different values of varactor capacitance. HFSS simulation results are denoted with crosses and circles for varactor capacitance values of $60 \mathrm{fF}$ and $120 \mathrm{fF}$, respectively. The simulation results for normal incidence, $45^{\circ}(\mathrm{TM})$, and $45^{\circ}$ (TE) are colored blue, green, and red, respectively.

\section{NUMERICAL VALIDATION AND RESULTS}

In this section the propagation properties of a parallel-plate waveguide having either one or two artificial impedance surfaces are studied analytically. The analytical results are verified with simulations using Ansoft's High Frequency Structure Simulator (HFSS).

The impedance surfaces for the waveguides are designed for the lower millimeter wave region, namely Ka-band (26-40 GHz). Following the notations in Fig. 2] the parameters of the studied artificial impedance surface are: $a=b=1 \mathrm{~mm}, w=0.1 \mathrm{~mm}, h=0.2 \mathrm{~mm}$, and $\varepsilon_{\mathrm{r}}=\varepsilon_{2}=4$. The medium inside the impedance waveguide is air $\left(\varepsilon_{1}=1\right)$. As in any resonant circuit, the bandwidth of the resonance can be increased by increasing the effective inductance (the height of the grounded dielectric slab) with respect to the effective capacitance. Here the resonance bandwidth of the high-impedance surface is decreased intentionally so that the effects due to the resonance are clearly recognizable in the dispersion plots. The varactors on each edge of the patch are modeled with lumped capacitive sheets whose value of capacitance is changed depending on the studied case. The simulation model of the high-impedance surface is shown in Fig. 4 The periodicity in the simulation model is achieved by using the periodical boundary conditions available in HFSS.

According to (14) the grid capacitance of the designed impedance surface is approximately $26 \mathrm{fF}$. Based on this information and knowing the frequency band of interest, the capacitance of the varactors is varied from $60 \mathrm{fF}$ to $120 \mathrm{fF}$; with these values the resonance frequency of the surface for the normal incidence appears to be approximately at $34 \mathrm{GHz}$ and $26 \mathrm{GHz}$, respectively. Furthermore, the capacitance of the varactors is considerably larger than the grid capacitance, as discussed earlier. 


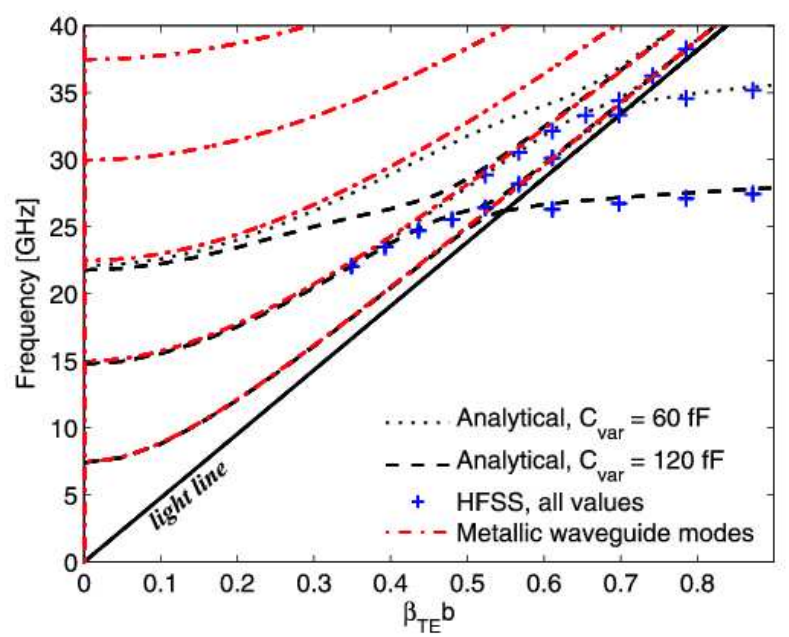

(a)

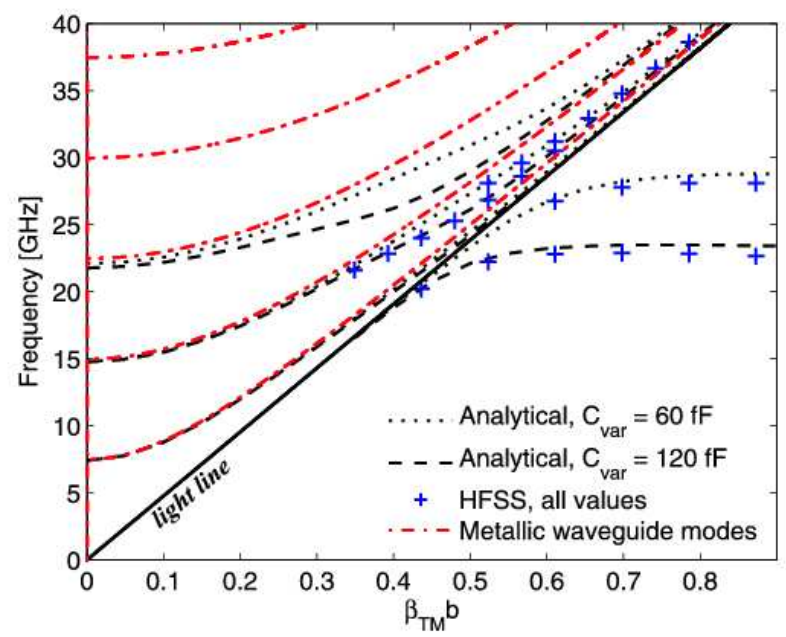

(b)

Fig. 6. Color online. (a) The propagation properties for TE modes in an impedance waveguide with one tunable impedance surface. (b) The propagation properties for TM modes in an impedance waveguide with one tunable impedance surface. The fundamental modes of metal waveguide are plotted with dash-dot lines. $\beta_{\mathrm{TE}}$ and $\beta_{\mathrm{TM}}$ refer here to the propagation constants of the TE and TM modes, respectively. Only the two lowest modes are simulated.

The reflection phases of the surface for different values of $C_{\text {var }}$ and for different incident angles are shown in Fig. 5. The analytical results concur with the simulation results very accurately. Clearly the bandwidth for the TE polarization becomes smaller and the bandwidth for the TM polarization larger as the angle of incidence grows. The effect of this to the propagation properties of the impedance waveguide will be discussed later.

\section{A. A multi-mode waveguide}

Before moving on to the interpretation of the dispersion plots, the terms used in this and following paragraphs need to be clarified. It is natural to use the modes propagating in an empty metallic parallelplate waveguide as reference cases when studying the propagation properties of an impedance waveguide. Therefore the modes propagating in a metallic waveguide will be referred as fundamental modes from here on in order to distinguish them from the modes of an impedance waveguide (referred plainly as modes). 


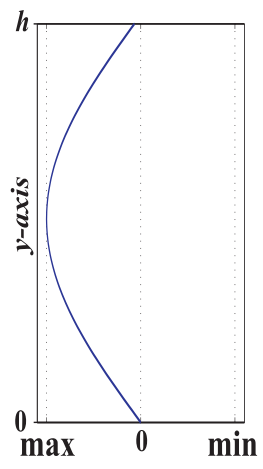

(a)

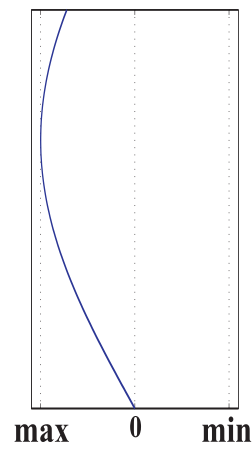

(b)

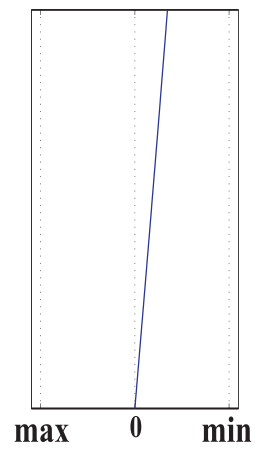

(c)

Fig. 7. The normalized magnitude of the $x$-component of the electric field for the $\mathrm{TE}_{01}$ mode at points (a) $\beta b=0.5, f=25.0 \mathrm{GHz}$ (b) $\beta b=0.69, f=33.5 \mathrm{GHz}$, and (c) $\beta b=0.71, f=34.0 \mathrm{GHz}$

The dispersion curves of a 20 -mm high parallel-plate waveguide with one tunable impedance surface are shown in Fig. 6. The second surface is perfectly conducting metal. The height of the waveguide was chosen so that many fundamental modes would propagate in the waveguide at Ka-band. The fundamental modes of a parallel-plate waveguide are shown with dash-dot lines. The simulation results have been marked by crosses in Fig. 6. The concurrence between the analytical and numerical results is very good.

In Fig. 6 near the resonance frequency of the impedance surface a $180^{\circ}$ mode hop is observed for both polarizations. The propagating wave exhibits a $180^{\circ}$ phase shift while the mode morphs from one fundamental mode to another. This is due to the change of the reflection phase of the impedance surface. For instance, a wave propagating at $27.5 \mathrm{GHz}$ near the second fundamental mode, $\mathrm{TE}_{02}$ in Fig. 6(a), morphs into the first fundamental mode, $\mathrm{TE}_{01}$, when the varactor capacitance is changed gradually from $60 \mathrm{fF}$ to $120 \mathrm{fF}$ over a certain distance. This is similar to the behavior of corrugated-waveguide mode converters, where the depth of the corrugations is tapered gradually [29]. Similar behavior occurs for higher-order modes and for $\mathrm{TM}_{01}$ as well. The field pattern of the $\mathrm{TE}_{01}$ mode is plotted in Fig. 7.

The mode conversion is seen to happen more gradually for the TM modes in Fig. 6 (b) than for the TE modes in Fig. 6 (a). This is because the bandwidth of the high-impedance surface becomes wider for TM polarized fields than for TE polarized fields as the angle of incidence grows, as discussed above. This creates an advantage for the TE modes over the TM modes in tunable impedance waveguide applications: The needed range of tuning of the resonant frequency of the high-impedance surface is smaller for the TE modes than for the TM modes in impedance waveguide applications.

The dispersion curve for a parallel-plate waveguide having two tunable impedance surfaces is shown in Fig. 8. Similar mode conversion is seen as in the case of just one impedance surface. However, instead of a $180^{\circ}$ mode hop discussed earlier, a $360^{\circ}$ mode hop occurs. This is simply because both impedance surfaces induce a $180^{\circ}$ mode hop.

In the case of two tunable impedance surfaces (see Fig. 8 (a)) interesting properties in the vicinity of the resonance frequency of the surface are seen: Both $\mathrm{TE}_{01}$ and $\mathrm{TE}_{02}$ modes cross the light line at only slightly different points. The $\mathrm{TE}_{01}$ mode crosses the light line at $\beta b=0.71, f=33.9 \mathrm{GHz}$ whereas the $\mathrm{TE}_{02}$ mode crosses it at $\beta b=0.77, f=34.2 \mathrm{GHz}$. The field patterns of the $\mathrm{TE}_{01}$ and $\mathrm{TE}_{02}$ modes are shown in Figs. 9 and 10, respectively, for the case when $C_{\text {var }}=60 \mathrm{pF}$. Clearly, the modes can be divided into symmetric and asymmetric modes which is not possible in the case of just one tunable impedance wall.

\section{B. A single-mode waveguide}

The dispersion curves of a 7-mm high waveguide having one tunable impedance-surface are shown in Fig. 11 (a) and in Fig. 11 (b) for a waveguide having two tunable impedance-surfaces. In a regular 


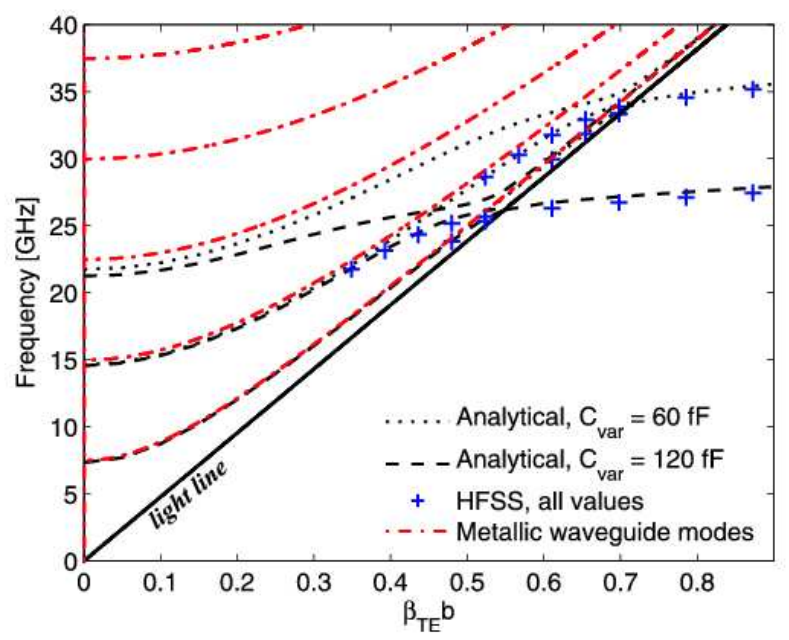

(a)

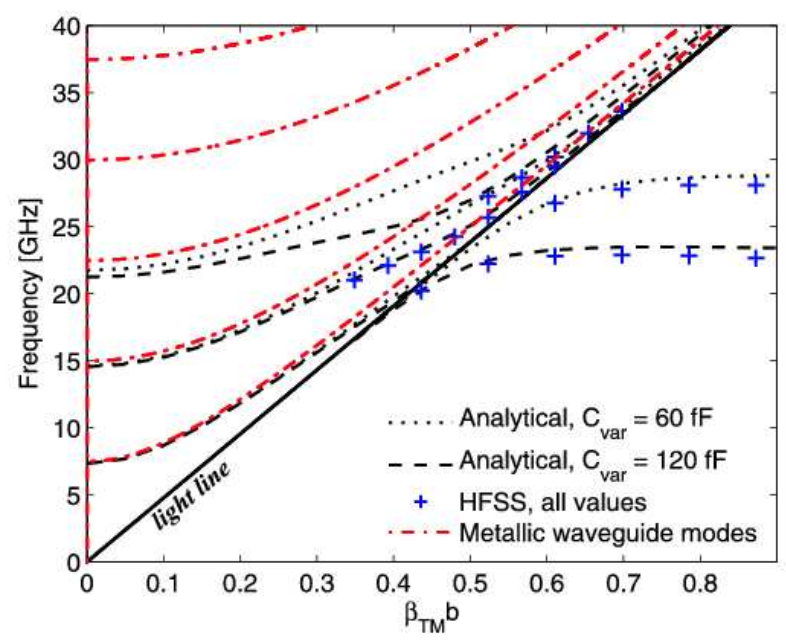

(b)

Fig. 8. Color online. (a) The propagation properties for TE modes in an impedance waveguide with two tunable impedance surfaces. (b) The propagation properties for TM modes in an impedance waveguide with two tunable impedance surfaces. The fundamental modes of metal waveguide are plotted with dash-dot lines. $\beta_{\mathrm{TE}}$ and $\beta_{\mathrm{TM}}$ refer here to the propagation constants of the TE and TM modes, respectively. Only the two lowest modes are simulated

metallic waveguide only one mode would propagate in the waveguide in the Ka-band. However, for the impedance waveguide, Fig. 11 clearly shows two (three) modes in the case of one (two) high-impedance sidewall(s). Because the response of the high-impedance surface for the normal incidence is the same for both TE and TM modes, also the cutoff frequencies are the same.

In Fig. 11 (a) the first TM mode diverges from the first fundamental mode and converges to the light line as we move through the resonance frequency of the high-impedance surface up to higher frequencies. The first TE mode diverges also from the first fundamental mode and crosses the light line at $f \approx 34 \mathrm{GHz}$ (the resonance frequency of the high-impedance surface having $C_{\mathrm{var}}=60 \mathrm{fF}$ ) after which the mode transforms into a surface-wave mode. The second mode in the waveguide has the cut-off frequency of $f=33.3 \mathrm{GHz}$. Both of these modes converge gradually to the first fundamental mode.

In the case of two impedance surfaces (Fig. 11 (b)) we can see two symmetric modes with cut-off frequencies of $19.7 \mathrm{GHz}$ and $35.3 \mathrm{GHz}$ and one asymmetric mode with a cut-off frequency of $31.6 \mathrm{GHz}$. Both the first symmetric and asymmetric TE modes cross the light line at $f=33.8 \mathrm{GHz}$ and $34.8 \mathrm{GHz}$, respectively, and transform into surface-wave modes. The first symmetric TM mode crosses the light line 




(a)

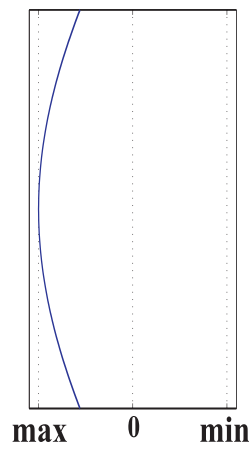

(b)

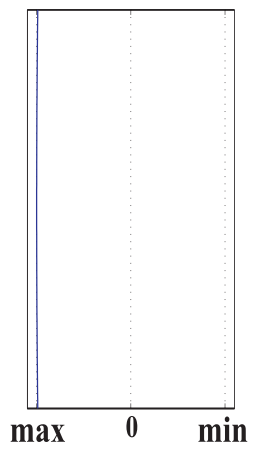

(c)

Fig. 9. The normalized magnitude of the $x$-component of the electric field for the $\mathrm{TE}_{01}$ mode at points (a) $\beta b=0.5, f=24.9 \mathrm{GHz}$ (b) $\beta b=0.69, f=33.3 \mathrm{GHz}$, and (c) $\beta b=0.71, f=33.9 \mathrm{GHz}$

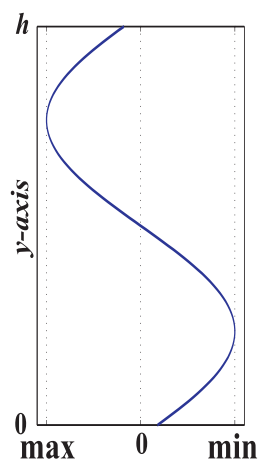

(a)

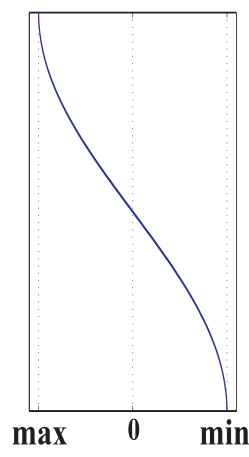

(b)

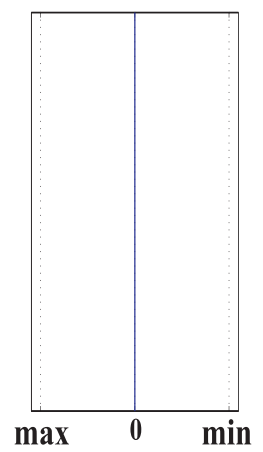

(c)

Fig. 10. The normalized magnitude of the $x$-component of the electric field for the $\mathrm{TE}_{02}$ mode at points (a) $\beta b=0.5, f=27.7 \mathrm{GHz}$ (b) $\beta b=0.69, f=33.9 \mathrm{GHz}$, and (c) $\beta b=0.77, f=34.2 \mathrm{GHz}$

at $f=27.8 \mathrm{GHz}$ and the second symmetric TM mode converges to the first fundamental mode. The asymmetric TM mode converges to the light line. This means that there exists a stop band for both TM and TE modes. For TE modes the width of the stop band is $1.5 \mathrm{GHz}$ between the symmetric TE waveguide modes and for the symmetric TM modes the width equals $7.5 \mathrm{GHz}$.

\section{A below-cutoff waveguide}

In Figs. 12 (a) and (b) the dispersion curves of a $3.5 \mathrm{~mm}$-high parallel-plate waveguide are shown for the cases of one and two impedance sidewalls, respectively. Fig. 12 shows that although no TE mode would propagate in the metallic waveguide below $42.8 \mathrm{GHz}$, one mode propagates in the impedance waveguide with one impedance sidewalls, and two modes propagate in the case of two impedance sidewalls. Similarly, in the case of TM modes, we find one or two extra modes below the cut-off frequency depending on the number of impedance sidewalls.

The TE modes form a narrow pass band both in the case of one and two impedance sidewalls. In the case of just one impedance sidewall the cutoff frequency of the first mode is $31.6 \mathrm{GHz}$ and the TE mode crosses the light line at $34.8 \mathrm{GHz}$. In the case of two impedance sidewalls the cutoff frequencies for the symmetric and the asymmetric modes equal $29.5 \mathrm{GHz}$ and $34.5 \mathrm{GHz}$, respectively. The symmetric mode crosses the light line at $33.9 \mathrm{GHZ}$ and the asymmetric mode crosses the light line at $35.8 \mathrm{GHz}$.

In the case of two impedance sidewalls both propagating TM modes demonstrate backward-wave propagation (see Fig. 13). The symmetric TM mode (cutoff frequency at $29.5 \mathrm{GHz}$ ) first propagates as a forward wave but transforms into a backward wave after the point $\beta d=0.4$. The asymmetric mode 


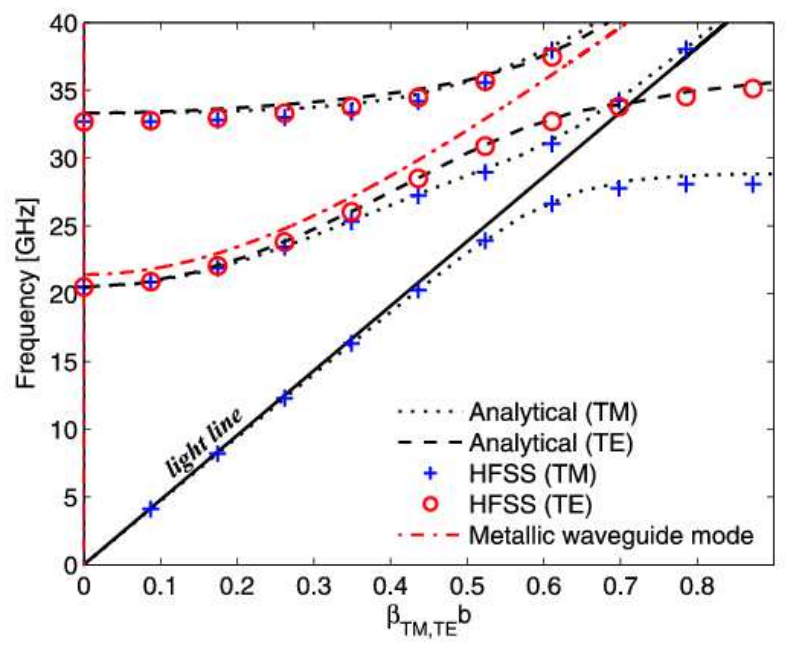

(a)

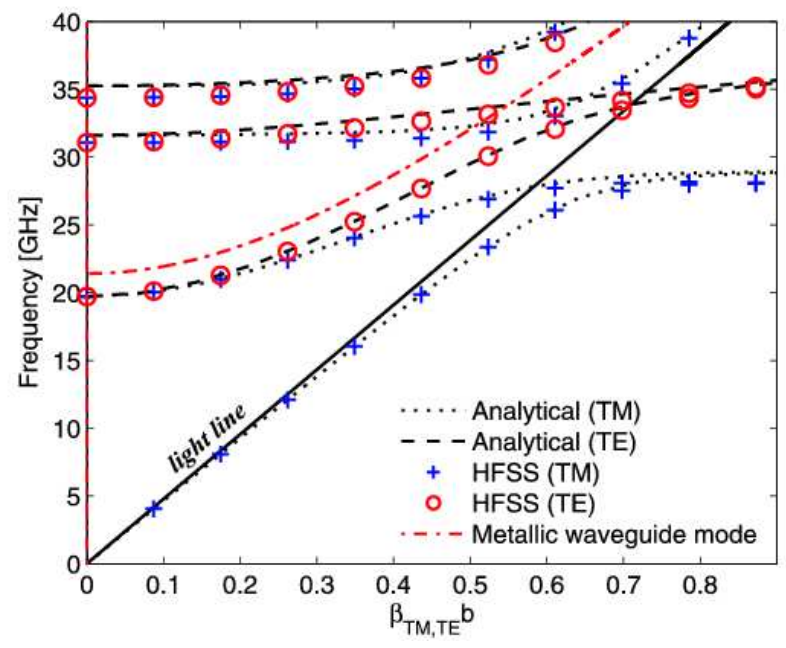

(b)

Fig. 11. Color online. The propagation properties of an 7-mm high impedance waveguide (a) with one tunable impedance surface and (b) with two tunable impedance surfaces. The value of the varactor capacitance is $C_{\mathrm{var}}=60 \mathrm{fF}$. The fundamental modes of metal waveguide are plotted with dash-dot lines. $\beta_{\mathrm{TE}}$ and $\beta_{\mathrm{TM}}$ refer here to the propagation constants of the TE and TM modes, respectively.

(cutoff frequency at $34.5 \mathrm{GHz}$ ) is first a backward wave and transforms to a forward wave after the point $\beta d=0.35$. In a $3.5-\mathrm{mm}$ metallic waveguide a TEM mode having the orientation of the field components similar to the considered TM mode would propagate. Figs. 12 (a) and (b) show that both with one or two tunable impedance surfaces it is possible to create a tunable stop band for the TEM mode.

\section{Discussion AND CONCLUSIONS}

An analytical model for a general type of tunable impedance surface comprising an array of patches has been presented. Dispersion equations for a parallel-plate waveguide having arbitrary surface impedance sidewalls have been presented. Together with the presented dispersion equations, the analytical model for the tunable high-impedance surfaces is used to study the propagation properties of impedance waveguide having either one or two tunable impedance surfaces. In oversized waveguide mode conversion is shown. In single-mode waveguide multi-mode propagation and band gaps are shown. Furthermore, forward- as well as backward-wave propagation in a below-cutoff waveguide is presented. The results are validated with commercial full-wave simulation software. The concurrence between the analytical and numerical results is very good. 




(a)

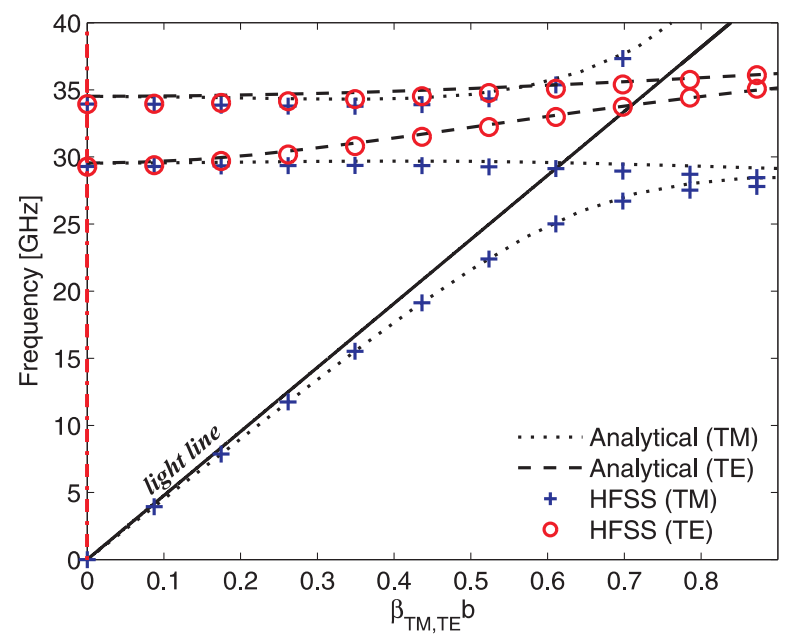

(b)

Fig. 12. Color online. (a) The propagation properties of a below-cutoff impedance waveguide with one tunable impedance surface. (b) The propagation properties of a below-cutoff impedance waveguide with two tunable impedance surfaces. The value of the varactor capacitance is $C_{\mathrm{var}}=60 \mathrm{fF}$. $\beta_{\mathrm{TE}}$ and $\beta_{\mathrm{TM}}$ refer here to the propagation constants of the TE and TM modes, respectively.

The presented analytical model for the study of the propagation properties in an impedance waveguide has proven to be very useful. The model has been verified and used to predict the dispersion in tunable impedance-wall waveguides in various example cases. The time needed to produce the results with the analytical model compared to the time consumed by numerical simulations is very marginal.

The dispersion results of the impedance waveguide show interesting features. In oversized waveguides tunable impedance surfaces allow one to realize tunable mode converters [29] and in many other application, where field transforming inside waveguides is needed. In addition, in single-mode or below the cutofffrequency waveguides possible applications for the tunable impedance surfaces include phase shifters [14], filters [16], [17], and lenses [15]. Without doubt, the design work for all these applications benefit from an efficient and simple model to predict the dispersion characteristics of an impedance waveguide. This list can be continued with such applications, as different types of antennas [12], [13], [30], tunable artificial magnetic conductors (AMC) [9], [31], and tunable electromagnetic band-gap structures (EBG) [9], [32], [33]. 


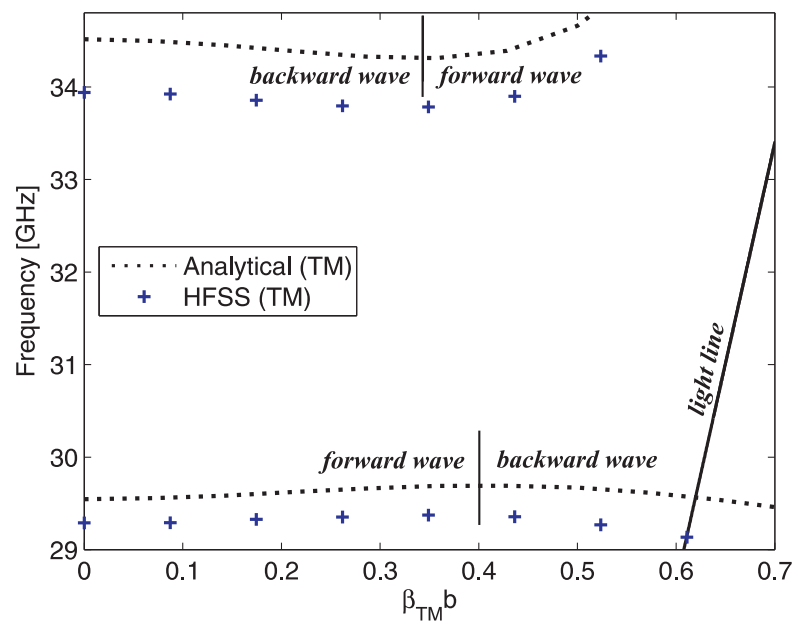

Fig. 13. A zoom-in of the Fig. 12. The backward wave propagation of the both symmetric and asymmetric TM modes.

\section{REFERENCES}

[1] P.-S. Kildal, "Artificially soft and hard surfaces in electreomagnetics," IEEE Transactions on Antennas and Propagation, vol. 38, no. 10, pp. 1537-1544, Oct. 1990.

[2] D. Sievenpiper, L. Zhang, R. F. J. Broas, N. G. Alexpolous, and E. Yablonovitch, "High-impedance electromagnetic surfaces with a forbidden frequency band," IEEE Transactions on Microwave Theory and Techniques, vol. 47, no. 11, pp. 2059-2074, Nov. 1999.

[3] R. E. Diaz, J. T. Aberle, and W. E. McKinzie, "TM mode analysis of a Sievenpiper high-impedance ractive surface," IEEE Antenna and Propagation International Symposium, vol. 1, pp. 327-330, July 2000.

[4] S. A. Tretyakov, and C. R. Simovski, "Dynamic model of artificial reactive impedance surfaces," Journal of Electromagnetic Waves and Applications, vol. 17, vo. 1, pp. 131-145, Nov. 2003.

[5] S. A. Tretyakov, Analytical Modeling in Applied Electromagnetics, Artech House, Inc., Norwood, MA, 2003.

[6] S. A. Tretyakov and S. I. Maslovski, "Thin absorbing structure for all incidence angles based on the use of a high-impedance surface," Microwave and Optical Technology Letters, vol. 38, no. 3, pp. 175-178, Jan. 2003.

[7] O. Luukkonen, C. Simovski, G. Granet, G. Goussetis, D. Lioubtchenko, A. V. Räisänen, and S. Tretyakov, "Simple and accurate analytical model of planar grids and high-impedance surfaces comprising metal strips or patches," to appear in IEEE Transactions on Antennas and Propagation, preprint available at http://arxiv.org/abs/0705.3548

[8] C. R. Simovski, P. de Maagt, S. A. Tretyakov, M. Paquay, and A. A. Sochava, "Angular stabilisation of resonant frequency of artificial magnetic conductors for TE-incidence," Electronics Letters, vol. 40, no. 2, pp. 92-93, Jan. 2004.

[9] G. Goussettis, A. P. Feresidis, and J. C. Vardaxoglou, "Tailoring the AMC and EBG characteristics of periodic metallic arrays printed on grounded dielectric substrate," IEEE Transactions on Antennas and Propagation, vol. 54, no. 1, pp. 82-89, Jan. 2006.

[10] S. Maci, M. Caiazzo, A. Cucini, and M. Casaletti, "A pole-zero mathcing method for EBG surfaces composed of a dipole FSS printed on a grounded dielectirc slab," IEEE Transactions on Antennas and Propagation, vol. 53, no. 1, pp. 70-81, Jan. 2005.

[11] F.-R. Yang, K.-P. Ma, Y. Qian, and T. Itoh, "A novel TEM waveguide using uniplanar compact photonic-bandgap (UC-PBG) structure," IEEE Transactions on Microwave Theory and Techniques, vol. 47, no. 11, pp. 2092-2098, Nov. 1999.

[12] D. F. Sievenpiper, J. H. Schaffner, H. J. Song, R. Y. Loo, and G. Tangonan, "Two-dimensional beam steering using an electrically tunable impedance surface," IEEE Transactions on Antennas and Propagation, vol. 51, no. 10, pp. 2713-2722, Oct. 2003.

[13] D. F. Sievenpiper, "Forward and backward leaky wave radiation with large effective aperture from an electronically tunable surface," IEEE Transactions on Antennas and Propagation, vol. 53, no. 1, pp. 236-247, Jan. 2005.

[14] J. A. Higgins, H. Xin, A. Sailer, and M. Rosker, "Ka-band waveguide phase shifter using tunable electromagnetic crystal sidewalls," IEEE Transactions on Microwave Theory and Techniques, vol. 51, no. 4, pp. 1281-1288, April 2003.

[15] H. Xin, J. B. West, J. C. Mather, J. P. Doane, J. A. Higgins, H. Kazemi, and M. J. Rosker, "A two-dimensional millimeter wave phase scanned lens utilizing analog electromagnetic crystal (EMXT) waveguide phase shifters," IEEE Transactions on Antennas and Propagation, vol. 53, no. 1, pp, 151-159, Jan. 2005.

[16] A. Higgins, H. Xin, and A. Sailer, "Tunable millimeter wave band pass filter using electromagnetic crystal sidewalls," in 2004 IEEE MTT-S Int. Microwave Symp. Dig., June 2004, pp. 1295-1298.

[17] H. Xin, A. Higgins, J. Hacker, M. Kim, and M. Rosker, "Electromagnetic crystal (EMXT) waveguide band-stopp filter," IEEE Microwave and Wireless Components Letters, vol. 13, no. 3, pp. 108-110, 2003.

[18] D. Chicherin, S. Dudorov, D. Lioubtchenko, V. Ovchinnikov, S. Tretyakov, and A. Räisänen, "MEMS-based high-impedance surfaces for millimeter and submillimeter wave applications," Microwave and Optical Technology Letters, vol. 48, no. 12, pp. 2570-2573, Dec. 2006.

[19] D. Sievenpiper, "High-impedance electromagnetic surfaces," Ph.D. Dissertation, Dept. Elect. Eng., Univ. California, Los Angeles, CA, 1999. 
[20] S. Clavijo, R. E. Díaz, and W. E. McKinzie, III, "Desing methodology for Sievenpiper high-impedance surfaces: An artificial magnetic conductor for positive gain electrically small antennas," IEEE Transactions on Antennas and Propagation, vol. 51, no. 10, pp. 26782690, Oct. 2003.

[21] J. M. Hao, L. Zhou, and C. T. Chan, "An effective medium model for high-impedance surfaces," Applied Physics A, vol. 87, no. 2, pp. 281-284, Jan. 2007.

[22] C. Mias and J. H. Yap, "A varactor-tunable high impedance surface with a resistive-lumped-element biasing grid," IEEE Transactions on Antennas and Propagation, vol. 55, no. 7, pp. 1955-1962, July 2007.

[23] J. A. Higgins, M. Kim, J. B. Hacker, and D. Sievenpiper, "The application of photonic crystals to quasi-optic amplifier," IEEE Transactions on Microwave Theory and Techniques, vol. 47, no. 11, pp. 2139-2143, Nov. 1999.

[24] E. Lier and P.-S. Kildal, "Soft and hard horn antennas," IEEE Transactions on Antennas and Propagation, vol. 36, no. 8, pp. 1152-1157, 1988.

[25] S. P. Skobelev and P.-S. Kildal, "Analysis of conical quasi-TEM horn with a hard corrugated section," IEEE Transactions on Antennas and Propagation, pt. I, vol. 51, no. 10, pp. 2723-2731, 2003.

[26] M. N. M. Kehn, M. Nannetti, A. Cucini, S. Maci, and P.-S. Kildal, "Analysis of dispersion in dipole-FSS loaded hard rectangular waveguide," IEEE Tranactions on Antennas and Propagation, vol. 54, no. 8, pp. 2275-2282, Aug. 2006.

[27] D. M. Pozar, Microwave Engineering, 2nd Edition, John Wiley \& Sons, Inc., New York, NY, 1998, pp. $109-110$.

[28] R. C. Compton, L. B. Whitbourn, and R. C. McPherdan, "Strip gratings at a dielectric interface and application of Babinet's principle," Applied Optics, vol. 23, no. 18, pp. 3236-3242, Sep. 1984.

[29] M. Thumm, "Computer-aided analysis and design of corrugated $\mathrm{TE}_{11}$ to $\mathrm{HE}_{11}$ mode converters in highly overmoded waveguides," International Journal of Infrared and Millimeter Waves, vol. 6, no. 7, pp. 577-597, 1985.

[30] S. V. Hum, M. Okoniewski, and R. J. Davies, "Modeling and desing of electrically tunable reflectarrays," IEEE Transactions on Antennas and Propagation, vol. 55, no. 8, pp. 2200-2210, 2007.

[31] A. P. Feresidis, G. Goussetis, S. Wang, and J. C. Vardaxoglou, "Artificial magnetic conductor surfaces and their application to low-profile high-gain planar antennas," IEEE Transactions on Antennas and Propagation, vol. 53, no. 1, pp. 209-215, 2005.

[32] A. P. Feresidis, G. Apostolopoulos, N. Serfas, and J. C. Vardaxoglou, "Closely coupled metallodielecric electrmagnetic band-gap structures formed by double-layer dipole and tripole arrays," IEEE Transactions on Antennas and Propagations, vol. 53, no. 5, pp. 11491158, 2004.

[33] F. Yang and Y. Rahmat-Samii, "Reflection phase characterizations of the EBG ground plane for low profile wire antenna applications," IEEE Transactions on Antennas and Propagation, vol. 51, no. 10, pp. 2691-2703, 2003. 